\title{
Sloshing effects in tanks containing liquid
}

\author{
Martin Sivý ${ }^{1, *}$, Miloš Musil ${ }^{1}$, Ondrej Chlebo ${ }^{1}$ and René Havelka ${ }^{1}$ \\ ${ }^{1}$ Institute of Applied Mechanics and Mechatronics, Faculty of Mechanical Engineering, \\ Slovak University of Technology, Námestie slobody 17, 81231 Bratislava, Slovakia
}

\begin{abstract}
Dynamic analysis of a tank containing liquid is a complex problem involving fluid-structure interaction. The tank-liquid system is simplified by an equivalent model in which the total liquid mass is divided into two zones - impulsive and convective. The paper is primary focused on the behavior of the free liquid surface (the convective portion of liquid) subjected to the dynamic loading which may result in liquid spilling or tank wall damage. Therefore, the sufficient freeboard must be required to design. The paper deals with the seismic design of the open cylindrical liquid storage tank with the aim to determine convective dynamic properties (natural frequencies and modes of oscillation), maximum vertical displacements over tank radius and overall response of the liquid to an earthquake. The analysis is performed analytically by applying procedures for the determination of convective effects based on simplified equivalent springmass model, numerically response spectrum, and method of motion integrating equations utilizing ANSYS Multiphysics.
\end{abstract}

\section{Introduction}

Cylindrical tanks or pressure vessels are commonly used in the power and processing industries. They can be employed as storage vessels for various liquids (from non-flammable, nontoxic liquids to highly volatile, toxic chemicals with explosive nature). The ordinary operation of tank-liquid systems may be influenced by seismic loading which can threaten the safety and integrity of these structures. During a seismic event, time-dependent hydrodynamic forces, pressures and stresses are induced by the liquid on the tank walls and bottom. Knowledge of hydrodynamic effects is essential since they influence the response of the tank and must be considered in the design. The procedures for evaluation of these effects are covered in international, national standards and/or guidelines (e.g. API 650, Eurocode 8).

Earthquake damage to steel storage tanks can come in several forms. One of the phenomena observed at the free liquid surface is a sloshing effect of the upper (convective) portion of the liquid. Oscillation of the convective liquid in the storage tanks may result in negative effects such as deformations and ruptures of the tank walls due to impact [1]. In addition, when open tanks are subjected to ground motions, sloshing may lead to liquid spillage. Therefore, the sufficient freeboard (clearance) between the free surface and the top of the tank or the roof must be provided.

\footnotetext{
* Corresponding author: martin.sivy@,stuba.sk
} 


\section{Basic concept}

Past earthquakes and their destructive consequences led to a huge effort to describe the response of the tank-liquid systems when subject to dynamic excitation resulting to provisions and recommendations for earthquake resistance to prevent negative effects to people, environment, operation, etc. Based on analytical and experimental studies of G. W. Housner [2], A. S. Veletsos [3], P. K. Malhotra [4] and others, procedures for the evaluation of seismic characteristics for liquid storage tanks were formulated.

In calculations of the seismic effects, simple equivalent mechanical models are used to investigated tank-liquid systems. The most widely used model adopted in various international codes (e.g. in [5]) is the one based on spring-mass modeling proposed for rigid tanks by G. W. Housner [2] which was later modified and extended for flexible tanks.

In the simplified model (Fig. 1a) the total liquid is divided into two parts - the impulsive liquid, located near the tank base which moves with (and deforms) the tank walls, and the convective liquid which represents sloshing of the free surface and oscillates independently of the tank wall. The impulsive portion of the liquid is represented by a respective mass $m_{\mathrm{i}}$ rigidly attached to the tank wall, whereas the convective part is described by an infinite number of masses $m_{\mathrm{c} n}$ flexibly attached by a spring of appropriate stiffness $k_{\mathrm{c} n}$. Each of the convective masses represents another slosh mode of the free liquid surface. In addition to spring-mass models, the convective portion of liquid can be replaced by another system, e.g. a system of simple pendulums (Fig. 1b), each of mass $m_{\mathrm{c} n}$ and length $l_{\mathrm{cn} n}$. All necessary parameters for the description of equivalent models can be found in $[5,6]$.

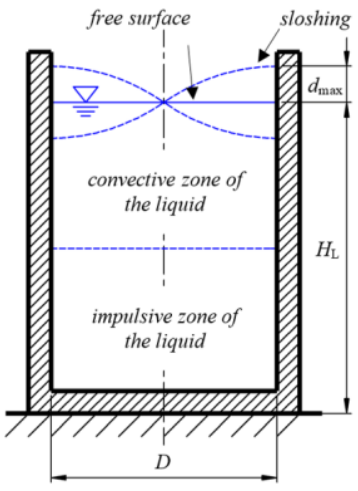

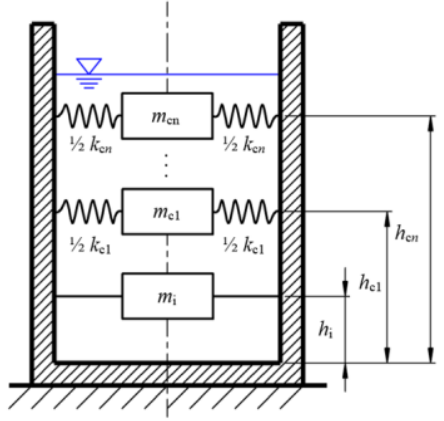

a)

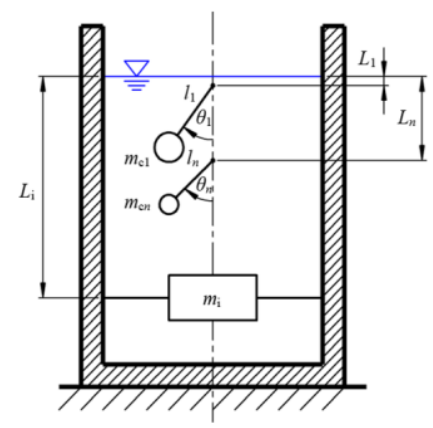

b)

Fig. 1. Equivalent simplified spring-mass and pendulum mechanical models.

\section{Liquid sloshing effects}

In this chapter, we will focus on the determination of dynamic properties (natural frequencies, modes of oscillation and sloshing wave height) analytically using procedures introduced in [3-5] and by seismic response of the convective liquid portion to earthquake load utilizing numerical analysis based on finite element method in ANSYS Multiphysics.

The investigated model is a flexible (steel) ground-supported liquid storage tank fixed to the rigid foundation of a circular cross-section of radius $R(5 \mathrm{~m})$, height $H(5 \mathrm{~m})$ and uniform wall thickness $t(0.005 \mathrm{~m})$. The tank is filled with water to a height of $H_{L}(4 \mathrm{~m})$.

In the FEM formulation, a tank is modeled by structural shell elements (SHELL181); liquid is represented by fluid elements based on Lagrange's formulation (FLUID80). At the interface between structural and fluid elements it is necessary to define the coupling of tank and liquid displacements. 


\subsection{Convective natural frequencies and modes of oscillation}

The motion of the convective portion of liquid can be expressed as a linear combination of the corresponding natural modes of oscillation. Each respective mode is associated with vertical displacements and is described analytically by the Helmholtz equation. The solution for each slosh mode of a circular liquid storage tank with radius $R$ is proportional to

$$
S_{n, m}=J_{n}\left(\lambda_{n, m} \frac{r}{R}\right) \cos n \theta
$$

which varies towards the circumference by $\cos n \theta$. $J_{n}$ represents a Bessel function of the first kind and $n^{\text {th }}$ order and $\lambda_{n, m}$ are values for which the first derivative of $J_{n}^{\prime}$ are zero.

The natural frequency of the convective portion of liquid for the slosh mode of oscillation can be calculated as

$$
f_{\mathrm{c}(n, m)}=\frac{1}{2 \pi} \sqrt{\lambda_{n, m} \frac{g}{R} \tanh \left(\lambda_{n, m} \frac{H_{\mathrm{L}}}{R}\right)}
$$

Using Eq. (1) and Eq. (2), it is possible to calculate any convective mode of oscillation at the free liquid surface and its respective natural frequency. In international standards, only the vertical displacements antisymmetric about the axis of rotation (one nodal diameter) can be calculated, i.e. the Bessel function of the first order is employed.

Fig. 2 presents the selected convective modes of oscillation for investigation of the tank model determined by Eq. (1) at frequencies calculated by Eq. (2). Based on results from seismic analyses of liquid storage tanks introduced in other publications of the authors, e.g. $[7,8]$, a good correlation of natural convective frequencies and modes of oscillation between analytical and numerical calculations (using FEM in ANSYS Multiphysics) is shown.
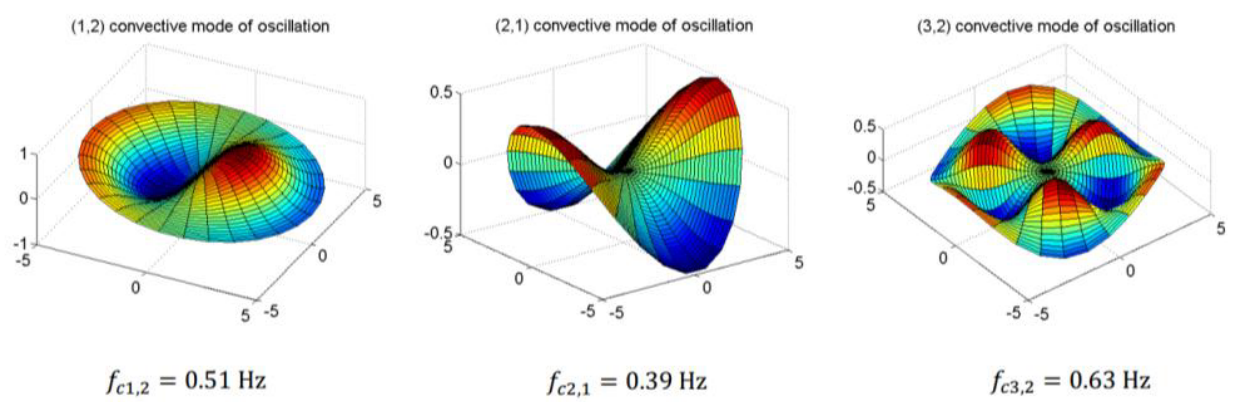

Fig. 2. Convective modes of oscillation and respective natural frequencies.

\subsection{Sloshing response to seismic excitation}

The natural frequencies of convective liquid are low in comparison with the impulsive portion of the liquid whose modes of oscillation are characterized by higher frequencies. Thus, the convective liquid is imparted to low acceleration but experiences high deformations, whereas impulsive liquid experiences high acceleration and low deformations during ground motion [9]. The convective liquid causes the sloshing motion and it does not contribute to a significant pressure component to the total hydrodynamic pressure.

For the seismic input, data recorded from the Parkfield California earthquake with a PGA of $4.25 \mathrm{~m} / \mathrm{s}^{2}$ at $7.52 \mathrm{~s}$ was used. Acceleration time-history of the earthquake and its response spectrum for proportional damping of $0.5 \%$ (liquid), $2 \%$ (steel or pre-stressed concrete structures) and 5\% (concrete and masonry structures) are presented in Fig. 3. 

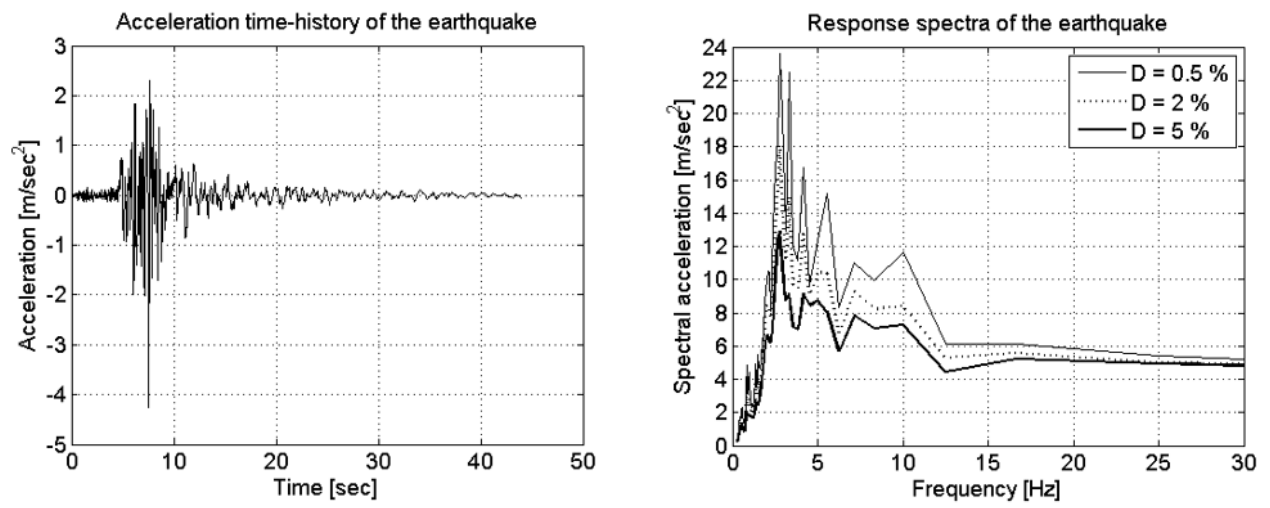

Fig. 3. Parkfield California earthquake.

\subsubsection{Sloshing wave height}

Introduced by A. S. Veletsos [3], the vertical displacement of free liquid surface due to sloshing may be analytically calculated from the following expression

$$
d(r, \theta, t)=R \sum_{n, m=1}^{\infty} \frac{2}{\lambda_{n, m}^{2}-1} \frac{J_{n}\left(\lambda_{n, m} \frac{r}{R}\right)}{J_{n}\left(\lambda_{n, m}\right)} \frac{s_{\mathrm{e}}\left(f_{\mathrm{c}(n, m))}\right.}{g} \cos n \theta
$$

where $S_{\mathrm{e}}\left(f_{\mathrm{c}}\right)$ is a convective spectral acceleration for a mode of oscillation corresponding to a natural frequency from a specific elastic response spectrum.

In codes aimed at tank-liquid systems design for earthquake resistance, the calculation of the sloshing wave height is reduced for specifying the maximum height at the edge by assuming only the first mode of oscillation. Fig. 4 presents the maximum vertical displacements of the tank model along the radius assuming only the $(1,1)$ natural mode. Using Eq. (3), the peak value of the sloshing wave at the edge is $0.37 \mathrm{~m}$.

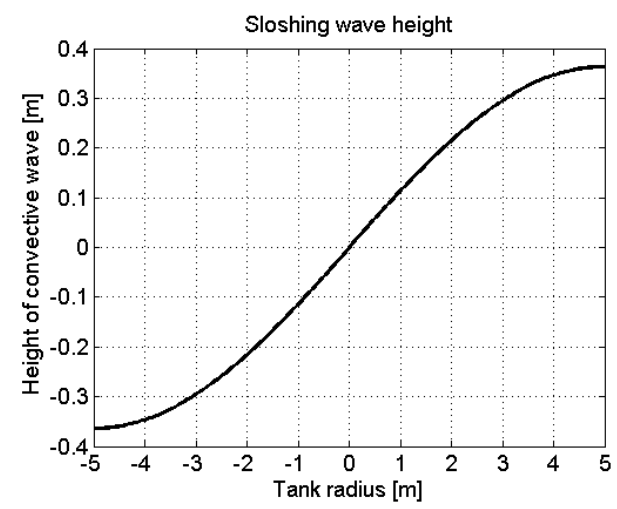

Fig. 4. Sloshing wave height.

\subsubsection{Response spectrum method}

One of the most common methodologies for describing the behavior of a linear structural system subject to seismic excitation is by performing a response spectrum modal analysis. It is often used instead of a time-history analysis to determine the response to time-dependent loading. 
The equation of motion for the MDOF system subject to earthquake ground acceleration $\ddot{u}_{\mathrm{g}}(t)$ is written as

$$
\mathbf{M} \ddot{\mathbf{u}}(t)+\mathbf{C} \dot{\mathbf{u}}(t)+\mathbf{K u}(t)=-\mathbf{M d} \ddot{u}_{\mathrm{g}}(t)
$$

where $\mathbf{M}, \mathbf{C}$ and $\mathbf{K}$ are mass, damping and stiffness matrices; $\mathbf{u}, \dot{\mathbf{u}}, \ddot{\mathbf{u}}$ are the displacement, velocity and acceleration vectors and $\mathbf{d}$ is the excitation direction vector (direction cosines). Using modal transformation

$$
\mathbf{u}(t)=\sum_{i=1}^{n} \boldsymbol{\phi}_{i} \mathrm{w}_{i}(t)
$$

multiplying by $\boldsymbol{\phi}_{j}^{\mathrm{T}}$ and application of appropriate orthogonality conditions, Eq. (4) can be written as several uncoupled equations of motion representing a set of SDOF systems

$$
\ddot{\mathrm{w}}_{i}(t)+2 \omega_{i} \xi_{i} \dot{\mathrm{w}}_{i}(t)+\omega_{i}^{2} \mathrm{w}_{i}(t)=-\gamma_{i} \ddot{\mathrm{u}}_{\mathrm{g}}(t)
$$

in which $\gamma_{i}$ represents a participation factor for a given excitation direction

$$
\gamma_{i}=\boldsymbol{\phi}_{i}^{\mathrm{T}} \mathbf{M d}
$$

This procedure is applied only when the time histories of all the modal displacement vectors in all the modes are known.

The overall relative displacement for each mode of oscillation can be expressed by

$$
\mathbf{u}_{i \max }=\frac{\gamma_{i} S_{\mathrm{A} i}}{\omega_{i}^{2}} \boldsymbol{\phi}_{i}
$$

where $S_{\mathrm{A} i}$ represents the spectral acceleration for the $i^{\text {th }}$ mode obtained from the response spectrum of a recorded earthquake. For evaluation of the overall response, individual responses are combined by a proper combination method (e.g., SRSS, GRP, CQC, etc.).

For computation of response of the free liquid surface, single-point response spectrum analysis is performed. The tank-liquid system is excited with a corresponding response spectrum for $0.5 \%$ proportional damping of the Parkfield California earthquake record (Fig. 3 ) in the $x$-direction. Based on results from the modal analysis, SRSS method is used for the combination of maximum modal responses. This combination rule is used due to closely spaced modes of oscillation. Fig. 5 presents the overall response of the tank-liquid system to the response spectrum. The maximum response of the liquid-structure system is $0.349 \mathrm{~m}$.
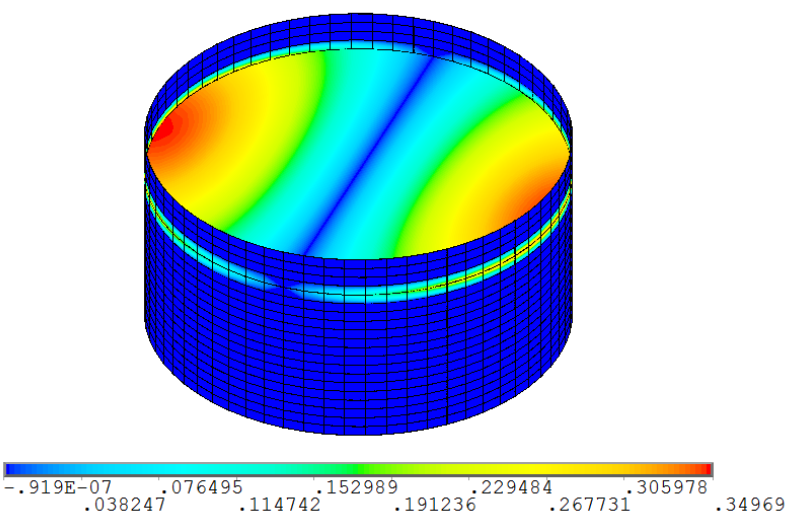

Fig. 5. Sloshing response of the tank corresponding to response spectrum. 


\subsubsection{Method of integrating of motion equations}

Integration of motion equations is a method used in a time-history analysis. This analysis is a universal technique used to determine the response of the structures subjected to any timedependent loading. The commonly used numerical integration method for evaluating the dynamic response of a structures is the Newmark's implicit method (more e.g. in $[10,11]$ ).

The system of equations of motion of a MDOF system is

$$
\mathbf{M} \ddot{\mathbf{u}}(t)+\mathbf{C} \dot{\mathbf{u}}(t)+\mathbf{K u}(t)=\mathbf{F}(t)
$$

where $\mathbf{F}(t)$ is the applied external load. In the case of the seismic excitation, the force $\mathbf{F}(t)$ is replaced in Eq. (9) by $-\mathbf{M} \ddot{u}_{g}(t)$.

Applying the Newmark's procedure, Eq. (9) is transformed into its static equivalent

$$
\widetilde{\mathbf{K}} \mathbf{u}_{t+\Delta t}=\widetilde{\mathbf{F}}_{t+\Delta t}
$$

where $\widetilde{\mathbf{K}}$ represents the effective stiffness and $\widetilde{\mathbf{F}}_{t+\Delta t}$ the effective load vector which are

$$
\begin{gathered}
\widetilde{\mathbf{K}}=\frac{1}{\alpha \Delta t^{2}} \mathbf{M}+\frac{\delta}{\alpha \Delta t} \mathbf{C}+\mathbf{K} \\
\tilde{\mathbf{F}}_{t+\Delta t}=\mathbf{F}_{t+\Delta t}+\mathbf{M}\left(\frac{1}{\alpha \Delta t^{2}} \mathbf{u}_{t}+\frac{1}{\alpha \Delta t} \dot{\mathbf{u}}_{t}+\left(\frac{1}{2 \alpha}-1\right) \ddot{\mathbf{u}}_{t}\right)+ \\
+\mathbf{C}\left(\frac{\delta}{\alpha \Delta t} \mathbf{u}_{t}+\left(\frac{\delta}{\alpha}-1\right) \dot{\mathbf{u}}_{t}+\frac{\Delta t}{2}\left(\frac{\delta}{\alpha}-2\right) \ddot{\mathbf{u}}_{t}\right)
\end{gathered}
$$

in which $\alpha, \delta$ represent Newmark's integration parameters which influence accuracy and stability of the algorithm (average acceleration method, linear acceleration method).

Eq. (10) can be easily solved. To achieve correct results, the choice of the integration time step is important and depends on the integration method. If seismic excitation is applied, the integration step should not be shorter than the interval of the input acceleration data [11].

To evaluate the time-dependent sloshing response of the convective liquid, the acceleration time-history of the Parkfield California earthquake (Fig. 3 ) in the $x$-direction is applied. Fig. 6 presents the maximum sloshing response of the tank storing water. This maximum vertical displacement of $0.3397 \mathrm{~m}$ occurs at the edge of the tank at time $10.16 \mathrm{sec}$.
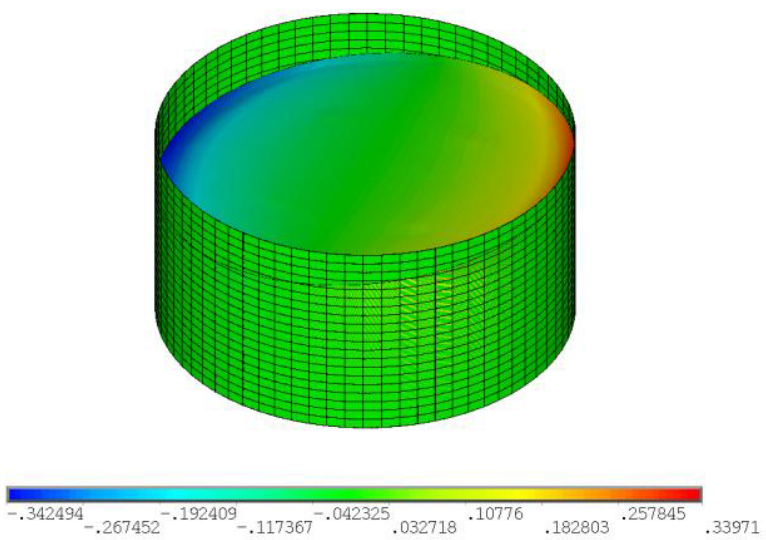

Fig. 6. Sloshing response of the tank to earthquake excitation. 


\section{Conclusions}

The intention of this contribution was to perform a seismic analysis of the liquid storage tank focusing on the sloshing response to a given earthquake loading. Generating of sloshing waves during ground motions represents unfavourable conditions which impacts the top and the walls within closed tanks or can result in the liquid spilling when open tanks are considered. A minimum freeboard is desired to prevent these negative effects. Insufficient freeboard leads to increase of impulsive mass due to the constraining action of the roof and upward load due to impacts generating the forces, which can break the connections between the tank and head. It may lead to buckling of the tank at the base if the loading from the additional impulsive mass was not considered in the design.

The convective response was determined analytical with respect to international standards, subsequently the response was calculated by FE software in ANSYS Multiphysics in which response spectrum and time-history analysis were performed. The results obtained between each solution of the dynamic analyses represented good conformity.

This work was supported by the grant from the Grant Agency of VEGA no. 1/0742/15 entitled Analysis for Seismic Resistance of Liquid Storage Tanks with Nonlinear and Time-Dependent Parameters, by the Slovak Research and Development Agency under the contract no. APVV-15-0630 Extension of the Validity of the Computation Standards for the Seismically Resistant Liquid Storage Tanks, in terms of Safety at NPPs and Other Industrial Areas and by the STU Grant scheme for Support of Young Researchers entitled Design and Optimization of the Measuring Equipment for Vibration Tests.

\section{References}

1. K. Malhotra, M. Eeri, Earthquake Spectra 21, 4 (2005)

2. G. W. Housner, Bulletin of the Seismological Society of America 47, 1 (1987)

3. A. S. Veletsos, Seismic Response and Design of Liquid Storage Tanks, Guidelines for the Seismic Design of Oil and Gas Pipeline Systems (Technical Council on Lifeline Earthquake Engineering ASCE, 1984)

4. P. K. Malhotra, T. Wenk, M. Wieland, Struct. Eng. Int. 10, 3 (2000)

5. EN 1998-4, Eurocode 8 - Design of Structures for Earthquakes Resistance, Part 4 Silos, Tanks and Pipelines (2006)

6. R. A. Ibrahim, Liquid Sloshing Dynamics Theory and Applications (Cambridge University Press, Cambridge, 2005)

7. M. Sivy, M. Musil, Procedure for Seismic Analysis of Liquid Storage Tanks using FEM Approach and Analytical Models, Advances in Mechanism Design II: Proceedings of the XII International Conference on the Theory of Machines and Mechanisms (Springer, Liberec, 2017)

8. M. Sivy, M. Musil, Stroj. cas. - J. of Mech. Eng. 66, 2 (2016)

9. P. K. Malhotra, P. Nimse, M. Meekins, Struct. Eng. Int. 24, 4 (2014)

10. A. K. Gupta, Response Spectrum Method in Seismic Analysis and Design of Structures (Blackwell Scientific Publications, Boston, 1990)

11. M. N. Fardis, E. C. Carvalho, P. Fajfar, A. Pecker, Seismic Design of Concrete Buildings to Eurocode 8 (CRC Press, Boca Raton, 2015) 